JASC 12-2-11

\title{
A Case Study of Green Ambience through Green Cloud Computing
}

\author{
*Rethina Kumar, Jeong-Jin Kang ${ }^{\dagger}$ \\ $\dagger^{* *}$ Dept. of Information and Communication, Dong Seoul University, Korea.
}

\begin{abstract}
Green cloud computing refers to the green ambient benefits that information technology services delivered over the Internet can offer for the society. The green meaning environment friendly and cloud computing is a traditional symbol for the Internet and a type of service provider. Cloud computing has drastically increased the number of datacenters and the energy consumption of data centers and that has become a critical issue which is extremely important in green ambience. These days the cloud data center needs high energy resources that leads to high operational cost and also maximizes CO2 - carbon footprint that pollutes the ambience which is not to be considered as green ambience. So we need to provide a way that leads us to green ambience. Cloud computing for the green ambience should be designed in a way which will utilize less energy resources and to minimize the $\mathrm{CO} 2$-carbon footprint, known as green cloud .In this paper we discuss various elements of Clouds which contributes to minimize the total energy consumption and the carbon emission so as to enable green ambience through green cloud computing .
\end{abstract}

Key words: green cloud computing, green data centers, green ambience

\section{INTRODUCTION}

This increasing demand in cloud computing can be accomplished only through large-scale datacenters, which needs very high energy resources to operate the servers and other infrastructure such as cooling, storage and network systems. Many internet companies such as Google, Amazon, eBay, and Yahoo are operating such huge datacenters around the world. The cloud computing is a subscription-oriented approach by providing access to scalable infrastructure and services on-demand. So that the users can store, access, and share any amount of information in Cloud. Cloud computing benefits such as on-demand computing resources, faster and cheaper software development capabilities at low cost. Cloud computing requires high energy resources to process this services by cloud data centers. According to IDC (International Data Corporation) report, the global IT Cloud services spending is estimated to increase from $\$ 16$ billion in 2008 to $\$ 42$ billion in 2012, representing a compound annual growth rate

Manuscript received : Sept. 08, 2012 / revised : Nov. 1, 2012

†Corresponding Author: iwit2000@gmail.com Tel: +82-2-407-7718, Fax: +82-2-407-7716,

Dept. of Information and Communication, Dong Seoul University, Korea.
(CAGR) of 27\%.Attracted by this growth prospects, Web-based companies (Amazon, eBay, Salesforce.com), hardware vendors (HP, IBM, Cisco), telecom providers (AT\&T, Verizon), software firms (EMC/VMware, Oracle/Sun, Microsoft) and others are all investing huge amount of capital in establishing Cloud datacenters. According to Google's earnings reports, the company has spent \$US1.9 billion on datacenters in 2006, and \$US2.4 billion 2007 .

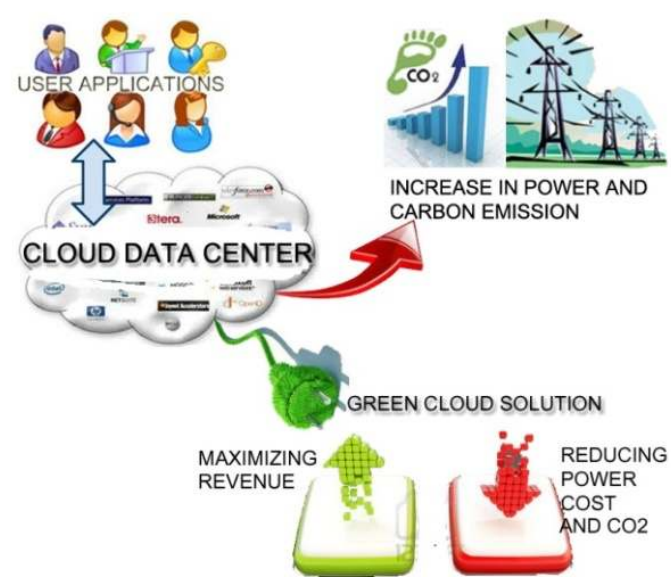

Fig. 1. Cloud Computing to Green Cloud Computing 
Clouds are essentially virtualized datacenters and applications offered as services on a subscription basis as shown in Figure 1. They require high energy usage for its operation, which leads to higher operational cost. For a datacenter, the energy cost is a significant component of its operating and up-front costs. In April 2007, Gartner estimated that the Information and Communication Technologies (ICT) industry generates about $2 \%$ of the total global $\mathrm{CO} 2$ emissions. According to a report published by the European Union, a decrease in emission volume of $15 \%-30 \%$ is required before year 2020 to keep the global temperature increase below $2{ }^{0} \mathrm{C}$. Thus, energy consumption and carbon emission by Cloud infrastructures has become a key environmental concern. Some studies show that Cloud computing can actually make traditional datacenters more energy efficient by using technologies such as resource virtualization and workload consolidation .A recent research by Accenture shows that moving business applications to Cloud can reduce carbon footprint of organizations. According to the report, small businesses saw the most dramatic reduction in emissions - up to 90 percent while using Cloud resources. Large corporations can save at least 30-60 percent in carbon emissions using Cloud applications, and mid-size businesses can save 60-90 percent.

The Greenpeace, observe that the Cloud phenomenon may aggravate the problem of carbon emissions and global warming. The reason given is that the collective demand for computing resources is expected to further increase dramatically in the next few years. Even the most efficiently built datacenter with the highest utilization rates will only mitigate, rather than eliminate, harmful $\mathrm{CO} 2$ emissions. The reason given is that Cloud providers are more interested in electricity cost reduction rather than carbon emission. The data collected by the study is presented Table 1 . Through the use of large shared virtualized datacenters Cloud computing can offer large energy savings.

Table 1. Comparison of Significant Cloud Datacenters

\begin{tabular}{|c|c|c|c|c|}
\hline $\begin{array}{c}\text { Cloud } \\
\text { data } \\
\text { centers }\end{array}$ & Location & $\begin{array}{c}\text { Estimated } \\
\text { power usage } \\
\text { Effectiveness }\end{array}$ & $\begin{array}{l}\text { \% of Dirty } \\
\text { Energy } \\
\text { Generation }\end{array}$ & $\begin{array}{c}\% \text { of } \\
\text { Renewable } \\
\text { Electricity }\end{array}$ \\
\hline Google & Lenoir & 1.21 & $\begin{array}{l}50.5 \% \text { Coal, } \\
38.7 \% \\
\text { Nuclear }\end{array}$ & $3.8 \%$ \\
\hline Apple & $\begin{array}{l}\text { Apple, } \\
\text { NC }\end{array}$ & & $\begin{array}{l}50.5 \% \text { Coal, } \\
38.7 \% \\
\text { Nuclear }\end{array}$ & $3.8 \%$ \\
\hline $\begin{array}{c}\text { Micros } \\
\text { oft }\end{array}$ & $\begin{array}{c}\text { Chicago, } \\
\text { IL }\end{array}$ & 1.22 & $\begin{array}{l}72.8 \% \text { Coal, } \\
22.3 \% \\
\text { Nuclear }\end{array}$ & $1.1 \%$ \\
\hline Yahoo & $\begin{array}{l}\text { La Vista, } \\
\quad \mathrm{NE}\end{array}$ & 1.16 & $\begin{array}{l}73.1 \% \text { Coal, } \\
14.6 \% \\
\text { Nuclear }\end{array}$ & $7 \%$ \\
\hline
\end{tabular}

However, Cloud services can also further increase the internet traffic and its growing information database which could decrease such energy savings. Thus, this chapter explores the environmental sustainability of Cloud computing by analyzing various technologies and mechanism that support this goal. Our analysis is important for users and organization that are looking at Cloud computing as a solution for their administrative, infrastructural and management problems.so we finally propose and recommend a Green Cloud framework to minimize the carbon footprint and minimize the energy resources without sacrificing the quality of service (performance, responsiveness and availability) offered by the multiple Cloud providers.

\section{RELATED RESEARCH}

Cloud computing is the delivery of computing as a service rather than a product, whereby shared resources, software, and information are provided to computers and other devices as a metered service over a network. Cloud computing is a marketing term for technologies that provide computation, software, data access, and storage services that do not require end-user knowledge of the physical location and configuration of the system that delivers the services. Cloud computing providers deliver applications via the internet, which are accessed from web browsers and desktop and mobile apps, while the business software and data are stored on servers at a remote location. While the computing resources are consolidated at a remote datacenter location.

\section{CLOUD COMPUTING}

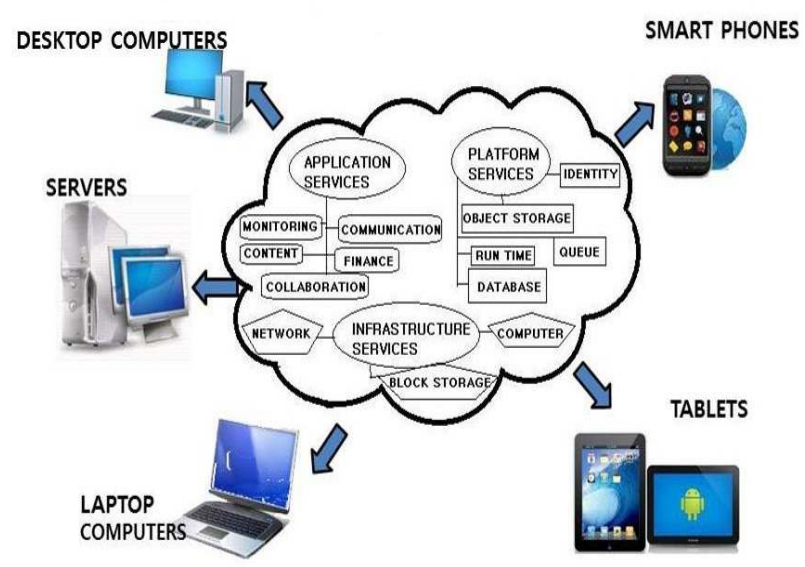

Fig. 2. cloud computing architecture

National Institute of Standards and Technology (NIST) defines Cloud computing as follows: "Cloud computing is a model for enabling convenient, on-demand network access to a shared pool of configurable computing resources (e.g., networks, servers, storage, applications, and services) that 
can be rapidly provisioned and released with minimal management effort or service provider interaction. This Cloud model promotes availability and is composed of five essential characteristics, three service models, and four deployment models."

\subsection{Characteristics of Cloud Computing}

The NIST simplified version of Cloud Computing:

- On-demand self-service: individuals can set themselves up without needing anyone's help;

- Ubiquitous network access: available through standard Internet-enabled devices;

- Location independent resource pooling: processing and storage demands are balanced across a common infrastructure with no particular resource assigned to any individual user;

- Rapid elasticity: consumers can increase or decrease capacity at will;

- Pay per use: consumers are charged fees based on their usage of a combination of computing power, bandwidth use and/or storage

\subsection{Cloud Computing Delivery models}

- Cloud Software as a Service (SaaS): Customers rent software hosted by the vendor;

- Cloud Platform as a Service (PaaS): Customers rent infrastructure and programming tools hosted by the vendor to create their own applications;

- Cloud Infrastructure as a Service (IaaS): Customers rent processing, storage, networking and other fundamental computing resources for all purposes.

\subsection{The possible deployment models}

- Private cloud: The cloud infrastructure is owned or leased by a single organization and is operated solely for that organization.

- Community cloud: The cloud infrastructure is shared by several organizations and supports a specific community that has shared concerns (e.g., mission, security requirements, policy, and compliance considerations).

- Public cloud: The cloud infrastructure is owned by an organization selling cloud services to the general public or to a large industry group.

- Hybrid cloud: The cloud infrastructure is a composition of two or more clouds (internal, community, or public) that remain unique entities but are bound together by standardized or proprietary technology).

\subsection{Datacenter}

The Cloud datacenters are quite different from traditional hosting facilities. A cloud datacenter could comprise of many hundreds or thousands of networked computers with their corresponding storage and networking subsystems, power distribution and conditioning equipment, and cooling infrastructures. Due to large number of equipment's, datacenters can consume massive energy consumption and emit large amount of carbon. According to 2007 report on computing datacenters by US Environmental Protection Agency (EPA), the datacenters in US consumed about 1.5\% of total energy, which costs about $\$ 4.5$ billon. This high usage also translates to very high carbon emissions which was estimated to be about 80-116 Metric Megatons each year. Table 2 lists equipment's typically used in datacenters with their contribution to energy consumption. It can be clearly observed that servers and storage systems are not the only infrastructure that consumes energy in the datacenter. In reality, the cooling equipment's consume equivalent amount of energy as the IT systems themselves.

Table 2. Percent of Power Consumption by Each Datacenter Device.

\begin{tabular}{|l|l|}
\hline $\begin{array}{l}\text { Cooling device (Chiller, Computer Room Air } \\
\text { Conditioning (CRAC)) }\end{array}$ & $33 \%+9 \%$ \\
\hline IT Equipment & $30 \%$ \\
\hline $\begin{array}{l}\text { Electrical Equipment's (UPS, Power } \\
\text { Distribution Units (PDUs), lighting) }\end{array}$ & $28 \%$ \\
\hline
\end{tabular}

Each of these devices needs to be designed and used efficiently while ensuring that their carbon footprint is reduced. A key factor in achieving the reduction in power consumption of a datacenter is to calculate how much energy is consumed in cooling and other overheads. Standard metrics Power Usage Effectiveness (PUE) .The PUE of a datacenter is defined as the ratio of the total power consumption of a facility (data or switching center) to the total power consumption of IT equipment (servers, storage, routers, etc.). PUE varies from datacenters depending on the place where datacenter is located and devices used in its construction.

\section{PROPOSED GREEN DATACENTER}

A green data center is a repository for the storage, management, and dissemination of data in which the mechanical, lighting, electrical and computer systems are designed for maximum energy efficiency and minimum environmental impact. The construction and operation of a green data center includes advanced technologies and strategies. Here are some examples:

- Minimizing the footprints of the buildings

- The use of low-emission building materials, carpets and 
paints

- Sustainable landscaping

- Waste recycling

- Installation of catalytic converters on backup generators

- The use of alternative energy technologies such as photovoltaic, heat pumps, and evaporative cooling

- The use of hybrid or electric company vehicles

To build energy efficient datacenter, several best practices has been proposed to improve efficiency of each device from electrical systems to processor level the smart construction of the datacenter and choosing of its location the datacenters are being constructed in such a way that electricity can be generated using renewable sources such as sun and wind Currently the datacenter location is decided based on their geographical features; climate, fibre-optic connectivity and access to a plentiful supply of affordable energy. Energy source is also seen mostly in terms of cost not carbon emissions. Datacenter is its cooling system that contributes to almost $1 / 3$ of total energy consumption. In datacenter cooling, two types of approaches are used: air and water based cooling systems. In both approaches, it is necessary that they directly cool the hot equipment rather than entire room area. Thus newer energy efficient cooling systems are proposed based on liquid cooling, Nano fluid-cooling systems, and in-server, in-rack, and in-row cooling by companies such as Spray Cool. Other than that, the outside temperature/climate can have direct impact on the energy requirement of cooling system. Some systems have been constructed where external cool air is used to remove heat from the datacenter. Datacenters power efficiency is addressed is on the deployment of new power efficient servers and processors. Low energy processors can reduce the power usage of IT systems in a great degree. Many new energy efficient server models are available currently in market from vendors such as AMD, Intel, and others; each of them offering good performance/watt system.

\section{ENABLING GREEN CLOUD COMPUTING}

Cloud computing can result in higher energy usage by the datacenters There are several technologies and concepts employed by Cloud providers to achieve better utilization and efficiency than traditional computing. Lower carbon emission is expected in Cloud computing due to highly energy efficient infrastructure and reduction in the IT infrastructure itself by multi-tenancy. The key driver technology for energy efficient Clouds is "Virtualization," which allows significant improvement in energy efficiency of Cloud providers Virtualization is the process of presenting a logical grouping or subset of computing resources so that they can be accessed in ways that give benefits over the original configuration. By consolidation of underutilized servers in the form of multiple virtual machines sharing same physical server at higher utilization, companies can gain high savings in the form of space, management, and energy.

According to Accenture Report there are following four key factors that have enabled the Cloud computing to lower energy usage and carbon emissions from ICT. Due to these Cloud features, organizations can reduce carbon emissions by at least $30 \%$ per user by moving their applications to the Cloud. These savings are driven by the high efficiency of large scale Cloud data centers.

4.1 Dynamic Provisioning: In traditional setting, datacenters and private infrastructure used to be maintained to fulfill worst case demand. Thus, IT companies end up deploying far more infrastructure than needed. There are various reasons for such over-provisioning: a) it is very difficult to predict the demand at a time; this is particularly true for Web applications and b) to guarantee availability of services and to maintain certain level of service quality to end users. One example of a Web service facing these problems is a Website for the Australian Open Tennis Championship. The Australian Open Website each year receives a significant spike in traffic during the tournament period. The increase in traffic can amount to over 100 times its typical volume (22 million visits in a couple of weeks). To handle such peak load during short period in a year, running hundreds of server throughout the year is not really energy efficient. Thus, the infrastructure provisioned with a conservative approach results in unutilized resources. Such scenarios can be readily managed by Cloud infrastructure. The virtual machines in a Cloud infrastructure can be live migrated to another host in case user application requires more resources. Cloud providers monitor and predict the demand and thus allocate resources according to demand. Those applications that require less number of resources can be consolidated on the same server. Thus, datacenters always maintain the active servers according to current demand, which results in low energy consumption than the conservative approach of over-provisioning.

4.2 Multi-tenancy: Using multi-tenancy approach, Cloud computing infrastructure reduces overall energy usage and associated carbon emissions. The SaaS providers serve multiple companies on same infrastructure and software. This approach is obviously more energy efficient than multiple copies of software installed on different infrastructure. Furthermore, businesses have highly variable demand patterns in general, and hence multi-tenancy on the same server allows the flattening of the overall peak 
demand which can minimize the need for extra infrastructure. The smaller fluctuation in demand results in better prediction and results in greater energy savings.

4.3 Server Utilization: In general, on-premise infrastructure run with very low utilization, sometimes it goes down up to 5 to 10 percent of average utilization. Using virtualization technologies, multiple applications can be hosted and executed on the same server in isolation, thus lead to utilization levels up to $70 \%$. Thus, it dramatically reduces the number of active servers. Even though high utilization of servers results in more power consumption, server running at higher utilization can process more workload with similar power usage.

4.4 Datacenter Efficiency: As already discussed, the power efficiency of datacenters has major impact on the total energy usage of Cloud computing. By using the most energy efficient technologies, Cloud providers can significantly improve the PUE of their datacenters. Today's state-of-the-art datacenter designs for large Cloud service providers can achieve PUE levels as low as 1.1 to 1.2 , which is about $40 \%$ more power efficiency than the traditional datacenters. The server design in the form of modular containers, water or air based cooling, or advanced power management through power supply optimization, are all approaches that have significantly improved PUE in datacenters. In addition, Cloud computing allows services to be moved between multiple datacenter which are running with better PUE values. This is achieved by using high speed network.

\section{MONITORING/METERING}

It is essential to construct power models that allow the system to know the energy consumed by a particular device, and how it can be reduced. To measure the unified efficiency of a datacenter and improve its' performance per-watt, the Green Grid has proposed two specific metrics known as the Power Usage Effectiveness (PUE) and Datacenter Infrastructure Efficiency (DciE) .

PUE $=$ Total Facility Power/IT Equipment Power

DciE $=1 /$ PUE $=$ IT Equipment Power/Total Facility Power x $100 \%$

Here, the Total Facility Power is defined as the power measured at the utility meter that is dedicated solely to the datacenter power. The IT Equipment Power is defined as the power consumed in the management, processing, and storage or routing of data within the datacenter.

PUE and DCIE are most common metrics designed to compare the efficiency of datacenters. For measuring and modeling the power usage of storage system, Researchers from IBM have proposed a scalable, enterprise storage modeling framework called STAMP.

\section{PROPOSED GREEN CLOUD COMPUTING}

We provide a solution to enable Green Cloud computing. We propose a Green Cloud Flowchart; the view of the green Cloud flowchart is given in Figure 3. The goal of this flow chart is to achieve green ambience through green Cloud computing.

In the Green Cloud flowchart, users submit their Green Cloud service requests through a new middleware Called Green cloud advisor that is used to identify the greenest Cloud provider who can provide the better green cloud service to the user's request without sacrificing the quality of service (QOS). A user service request can be of three types i.e., software, platform or infrastructure. The Cloud Service providers can register their green data's like energy parameters for various services(SaaS,PaaS,IaaS),CO2 Emission ,PUE ,Network cost ,rate of electricity ,price tag etc.. to the green cloud advisor directory which is accessed by the Green Cloud Advisor. The Green Cloud Advisor is to identify green cloud services, pricing and time when it should be accessed for least carbon emission. The Green Cloud Advisor gets the current status of energy parameters for using various Cloud services from Carbon Emission Directory. The Carbon Emission Directory maintains all the data related to energy efficiency of Cloud service. This data may include PUE and cooling efficiency of Cloud datacenter which is providing the service, the network cost and carbon emission rate of electricity. The Green Cloud Advisor calculates the carbon emission of all the Cloud providers who are offering the requested Green Cloud service. Then, it identifies the set of services that will result in least carbon emission and buy these services on behalf users. The Green Cloud flowchart is designed such that it keeps track of overall energy usage of serving a user request. It depends on the two main components Carbon Emission Directory and Green Cloud Advisor Directory, which keeps track of energy efficiency of each Cloud providers and also gives incentive to the Cloud providers to make their service Green. From user side, the Green Cloud Advisor plays a crucial role in monitoring and selecting the Cloud services based on the user QoS requirements and ensuring minimum carbon emission for serving a user. Finally, the user acknowledges the green service and the feedback is stored in the green cloud advisor directory to improve the green cloud service. 


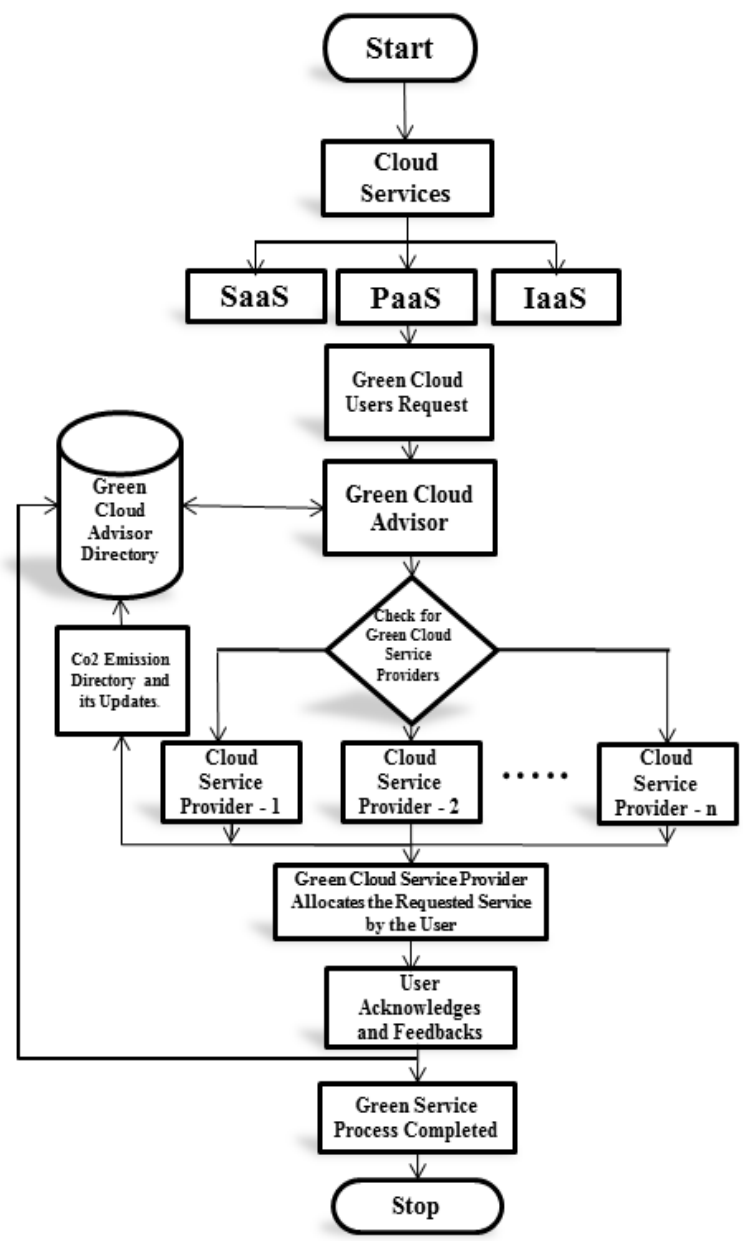

Fig. 3. Green Cloud Computing Flowchart

\section{CONCLUSION}

Cloud computing is one of the growing high demand services that is provided by information and communication technology (ICT) and it is considered as green for the environment. The Green IT analysis reports states that Clouds and datacenters are "Green", but other reports states that the increase in number of huge datacenters will leads to increase in Carbon emission. The carbon emission from data centers will triple between 2002 and 2020 which is currently 7.8 billion tons of $\mathrm{CO} 2$ per year. In this paper we have explained about the cloud computing and its benefits. we have tried to analyze the source that will help to achieve green ambiance through cloud computing. We have proposed a Green Cloud process and designed a flowchart that represents a way to achieve green cloud computing. In conclusion, by simply improving the efficiency of equipment, Cloud computing cannot be claimed to be Green. The main goal is to reduce the carbon footprint that harms the ambient and to make use of renewable energy sources from both user and provider's perspective.

\section{REFERENCES}

[1] Gleeson, E. 2009. Computing industry set for a shocking change. Retrieved January 10, 2010 from http://www.moneyweek.com/investment-advice/computing-i ndustry-set-for-a-shocking-change-43226.aspx

[2] New Datacenter Locations. 2008. http://royal.pingdom. com/2008/04/11/map-of-all-google-data-center-locations/

[3] Bianchini, R., and Rajamony, R. 2004, Power and energy management for server systems, Computer, 37 (11) 68-74.

[4] Rivoire, S., Shah, M. A., Ranganathan, P., and Kozyrakis, C. 2007. Joulesort: a balanced energy-efficiency benchmark, Proceedings of the 2007 ACM SIGMOD International Conference on Management of Data, NY, USA.

[5] Greenpeace International. 2010. Make IT Green http:// www.greenpeace.org/ international/en/publications/ reports/make-it-green-Cloudcomputing/

[6] Accenture Microsoft Report. 2010. Cloud computing and Sustainability: The Environmental Benefits of Moving to the Cloud, http://www.wspenvironmental.com/media /docs/newsroom/Cloud_computing_and_Sustainability_-_W hitepaper_-_Nov_2010.pdf.

[7] Mell, P. and Grance, T. 2009. The NIST Definition of Cloud computing, National Institute of Standards and Technology.

[8] Ranganathan P, 2010, Recipe for efficiency: principles of power-aware computing. Communication. ACM, 53(4):60-67.

[9] Rawson, A., Pledger, J., and Cadre, T., 2008. Green Grid Data Center Power Efficiency Metrics. Consortium Green Grid.

[10] Smith, J. and Nair, R. 2003. Virtual Machines: Versatile Platforms for Systems and Processes. Morgan Kaufmann: Los Altos, CA.

[11] IBM. 2008. Take the tennis to 1.9 billion viewers worldwide.

[12] Moore, J. D., Chase, J. S., and Ranganathan, P. 2006. Weatherman: Automated, online and predictive thermal mapping and management for datacenters, Proceedings of the 3rd International Conference on Autonomic computing, Dublin, Ireland.

[13] Woods, A. 2010. Cooling the data center. Communications of the ACM, 53(4):36-42.

[14] Allalouf, M., Arbitman, Y., Factor, M., Kat, R. I., Meth, K., and Naor, D. 2009. Storage modelling for power estimation. In Proceedings of 2009 Israeli Experimental Systems Conference (SYSTOR '09), Isreal. 


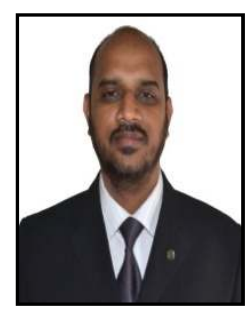

B.RETHINA KUMAR was born in India. He received his B.E.in Computer Science and Engineering and M.E. in Computer and Communication Engineering from India. Since 2001 he worked as a Lecturer, Senior Lecturer in the Department of Computer Science and Engineering and Department of Information Technology in Indian varsity. Since 2007 he is working as an Assistant Professor in Dong Seoul college, Korea in the Department of Information and communication engineering. His research areas of interests are Cloud Testing, Green Cloud Computing, Information Security and Information broadcast policies.

Mr. B.Rethina Kumar is a Member of the Institute of Webcasting, Internet and Telecommunication (IWIT) of Korea.

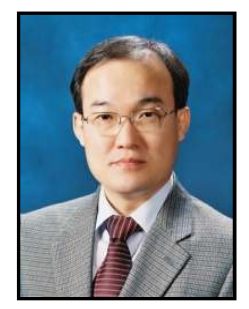

Jeong Jin Kang is currently the faculty of the Department of Information and Communication at Dong Seoul University in SeongNam, Korea since 1991, and currently the President of the Institute of Webcasting, Internet and Telecommunication(IWIT).

During 3 years from Feb. 2007 to Feb. 2010, he worked as a Visiting Professor at the Department of Electrical and Computer Engineering, The Michigan State University. He was a lecturer of the Department of Electronic Engineering at (Under)Graduate School(1991-2005), The Konkuk University.

Dr. Kang is a member of the IEEE Antennas and Propagation Society(IEEE AP-S), the IEEE Microwave Theory and Techniques Society(IEEE MTT-S), and a member of the Institute of Webcasting, Internet and Telecommunication(IWIT), Korea. His research interests involve Smart Mobile Electronics, RF Mobile Communication, Smart Convergence of Science and Technology, RFID/USN and $\mathrm{u}$-Healthcare, as well as New Media Service. 